\title{
INTERAÇÃO DO TRYPANOSOMA CRUZI COM DIFERENTES VETORES: USO PARA O XENODIAGNÓSTICO
}

\author{
Nelson J. Alvarenga e Elizabeth Bronfen
}

Foram estudadas as diferenças de interação (diferenciação e multiplicação) das cepas Ye CL de Trypanosoma cruzi, consideradas "polares", junto a Triatoma infestans, Panstrongylys megistus $e$ Dipetalogaster maximus.

Concluiu-se que o $\mathrm{T}$. infestans apresenta em suas populaçôes cerca de $40 \%$ de indivíduos com acentuada resistência à infecção pela cepa $Y$. $O \mathrm{P}$. megistus foi considerado um bom vetor onde se verificou pequena perda de infecção para ambas as cepas e boa diferenciação.

Melhor desenvolvimento e diferenciação para ambas as cepas foi observado em D. maximus e daí sua indicação para xenodiagnósticos.

Os autores concluem pela possibilidade da presença de fatores de resistência à infecção e também de inibidores de diferenciação como responsáveis por uma boa ou má interą̧ão parasito-vetor.

Palavras chaves: Interação. T. cruzi-vetor. Xenodiagnóstico. Fator de resistência à infecção. Diferenciação.

Diferentes autores têm se preocupado em determinar o vetor ideal para o $T$. cruzi e sua aplicação em xenodiagnóstico da doença de Chagas. A crença de que as especies locais de triatomineos são melhores adaptadas às cepas do $T$. cruzi também locais, foi motivo para elaboração de trabalhos onde se procurou esta confirmação (Dias6 ${ }^{6}$ Urdaneta-Morales e Rueda ${ }^{18}$ ).

Recentemente Perlowagora-Szumlewicz e cols $^{12}$ demonstraram uma variação da suscetibilidade à infecção por diferentes espécies de triatomíneos frente à cepa $\mathrm{Y}$ do $T$. cruzi.

No presente trabalho estudou-se em espécies de triatomineos provenientes de habitats diferentes a capacidade de manutenção de infecção e de metaciclogènese de duas cepas, $\mathrm{Y}$ e $\mathrm{CL}$, consideradas como "polares" por Melo 9 e Brener ${ }^{4}$.

\section{MATERIAL E MÉTODOS}

\section{Parasitas}

Foram utilizadas as cepas $\mathrm{Y}$ e CL do $T$. cruzi mantidas em laboratório por passagens periódicas em

Centro de Pesquisas Rene Rachou, FIOCRUZ, CP: 1743 30.000 Belo Horizonte - MG. Brasil.

Recebico para publicação em $3 / 8 / 83$. camundongos por inóculo de formas sangüineas. A cepa $\mathrm{Y}$, isolada a partir de caso humano por Silva e Nussenzweig ${ }^{11}$, no Estado de São Paulo e CL, isolada por Brener e Chiari ${ }^{5}$, a partir de $T$. infestans, naturalmente infectado, proveniente do Estado do Rio Grande do Sul.

\section{Insetos}

Foram utilizadas ninfas de $3 \%$ estadio de $T$. infestans e $P$. megistus e ninfas de 1 \% e 3 ? estágios de D. maximus.

Os insetos foram infectados por alimentação em camundongos apresentando parasitemia patente tendo sidos previamente anestesiados com Thionembutal.

Dependendo do objetivo desejado, procedia-se à contagem de parasitas circulantes no sangue dos camundongos em câmara de Neubauer e nestes casos os triatomíneos eram pesados antes e após o repasto infectante para se calcular o número de flagelados ingeridos por cada inseto.

Os triatomineos foram mantidos em condições ambientais ou em estufa em temperatura de $26-27^{\circ} \mathrm{C}$, sendo alimentados a cada 20 dias.

Os diversos exames para verificação da positividade de infecção dos triatomineos, foram feitos 
Alvarenga NJ, Bronfen E. Interação do Trypanosoma cruzi com diferentes vetores: uso para o xenodiagnóstico. Revista da Sociedade Brasileira de Medicina Tropical 17: 145-149, Jul-Set, 1984

individualmente seja por pressão abdominal, coleta de urina ou dissecção e retirada dos intestinos, conforme o objetivo do experimento. As contagens e a diferenciação das formas flageladas eram feitas em câmara de Neubauer após maceramento e suspensão em $1 \mathrm{ml}$ de PBS ( $\mathrm{pH}$ 7.2) de "pool" dos intestinos dos insetos sabidamente infectados.

\section{Avaliação estatística}

Utilizou-se o teste de diferença entre duas proporções comparando-se o percentual de insetos que apresentavam-se positivos e teste do $\mathrm{X}^{2}$.

\section{RESULTADOS}

$T$. infestans infectados pela cepa $\mathrm{Y}$ e mantidos em condiçōes ambientais por 60 dias apresentaram perda de infecção em $33 \%$ dos insetos, diferindo bastante dos resultados obtidos em $P$. megistus e D. maximus (Tabela I).

Tabela 1-Observação, por dissecção, da manutenção e perda de infecção em três espécies de triatomineos infectados pela cepa $Y$ do $T$. cruzi e mantidas em condicões ambientais por 60 dias.

\begin{tabular}{lcccrc}
\hline Espécie & Número & \multicolumn{2}{c}{ Positivos(\%) } & \multicolumn{2}{c}{ Negativos(\%) } \\
\hline T. infestans & 58 & 40 & $(67)^{*}$ & 18 & $(33)$ \\
D. maximus & 70 & 67 & $(96)$ & 3 & $(4)$ \\
$P$. megistus & 58 & 57 & $(98)$ & 1 & $(2)$ \\
\hline
\end{tabular}

(*) Diferença significativa para $\propto=0.05 \%$.

Um acompanhamento entre os 60 a 150 dias de T. infestans infectados pelas cepas $\mathrm{Y}$ e CL mostrou que sua perda de infecção apresenta indices relativamente constantes para ambas as cepas ( $\mathrm{Ta}$ bela 2). O tratamento estatístico mostrou diferença de proporção de infecção somente aos 60 dias, não se considerando diferentes os dados observados aos 90 , 120 e 150 dias.

O estudo das populações de flagelados obtidos de intestinos de $P$. megistus e $D$. maximus infectados pelas duas cepas demonstrou que ambas as espécies de triatomíneos se infectam bem. Considerando-se o percentual de obtenção de tripomastigotas metaciclicos nota-se um melhor rendimento por parte de D. maximus assim como também uma população muito maior de epimastigotas (Tabela 3 ).
Tabela 2 - Manutenção e perda de infecçâo de T. infestans infectados pelas cepas $Y$ e $C L$ do $T$. cruzi mantidos em condicóes ambientais e observados por disseç̧ão em diferentes periodos pós-infecção.

\begin{tabular}{lrlrlll}
\hline Dias & Cepa & Número & \multicolumn{3}{c}{ Positivos(\%) } & \multicolumn{2}{c}{ Negativos(\%) } \\
\hline \multirow{2}{*}{$60^{*}$} & Y & 20 & 13 & $(65)$ & 7 & $(35)$ \\
& CL & 20 & 20 & $(100)$ & - & \\
90 & Y & 24 & 16 & $(66.6)$ & 8 & $(33.3)$ \\
& CL & 20 & 18 & $(90)$ & 2 & $(20)$ \\
120 & Y & 12 & 8 & $(66.6)$ & 4 & $(33.3)$ \\
& CL & 12 & 11 & $(91.6)$ & 1 & $(8.33)$ \\
150 & Y & 15 & 9 & $(60)$ & 6 & $(40)$ \\
& CL & 15 & 13 & $(85)$ & 2 & $(15)$ \\
\hline
\end{tabular}

$\left(^{*}\right)$ Diferença estatisticamente significativa somente aos 60 dias.

Tabela 3 - Número de flagelados observados no trato digestivo de $\mathrm{P}$. megistus $e \mathrm{D}$. maximus infectados pelas cepas Ye CL do T. cruzie mantidos a $27^{\circ} \mathrm{C}$ por 60 dias.

\begin{tabular}{llrrrr} 
Espécie & Número Cepa & \multicolumn{2}{c}{$\begin{array}{c}\text { Metaciclicos/ } \\
\text { mililitro (\%) }\end{array}$} & $\begin{array}{c}\text { Epimastigotas } \\
\text { mililitro }\end{array}$ \\
\hline & & & & & \\
P. megistus & 20 & $\mathrm{Y}$ & 50.000 & $(3.9)$ & 1.280 .000 \\
& 20 & $\mathrm{CL}$ & 100.000 & $(4.1)$ & 2.400 .000 \\
& 20 & $\mathrm{Y}$ & $840.000^{*}$ & $(11.4)$ & 7.360 .000 \\
D. maximus & 20 & $\mathrm{CL}$ & $900.000^{*}$ & $(15.5)$ & 5.800 .000 \\
\hline
\end{tabular}

(*) Valores estatisticamente significativos: $\mathrm{X}^{2} \operatorname{lgl} \mathrm{p}<0.05$.

Tomando-se somente a metaciclogenese como parâmetro para medida de melhores condiçōes de adaptação do $T$. cruzi observa-se, em $T$. infestans e D. maximus, infectados pela cepa $\mathrm{Y}$ e examinados aos $30,60 \mathrm{e} 90$ dias, que o número de flagelados obtidos de $D$. maximus é muito superior ao de $T$. infestans chegando-se a obter um numero de metacíclicos 13,5 vezes maior na primeira espécie (Tabela 4). Os resultados mostrados nas Tabelas 3 e 4, analisados atraves do teste do $\mathrm{X}^{2}$ com $1 \mathrm{~g} 1$ para $\mathrm{p}<0,05$ demonstraram ser significativos a favor das infecções encontradas em D. maximus.

Para se verificar se o número de metacíclicos presentes nos intestinos dos insetos teria relação com o número de formas sangüineas ingeridas no momento da infecção, foram examinadas ninfas de 1. e 3 ? estádios de $D$. maximus ( 20 de cada) infectadas pela cepa $\mathrm{Y}$ e mantidas por 60 dias nas mesmas condições. 
Alvarenga NJ, Bronfen E. Interaçào do Trypanosoma cruzi com diferentes vetores: uso para o xenodiagnóstico. Revista da Sociedade Brasileira de Medicina Tropical 17: 145-149, Jul-Set, 1984

Tabela 4-Número de flagelados observados no trato digestivo de $\mathrm{T}$. infestans $e \mathrm{D}$. maximus infectados pela cepa $Y$ do $\mathrm{T}$. cruzi $e$ mantidos por diferentes periodos em temperatura ambiente.

\begin{tabular}{|c|c|c|c|c|}
\hline Dias & Espécie & Número & $\begin{array}{l}\text { Metacíclicos } \\
\text { mililitro (\%) }\end{array}$ & $\begin{array}{c}\text { Epimastigotas } \\
\text { mililitro }\end{array}$ \\
\hline \multirow[b]{2}{*}{30} & T. infe & 20 & 5.000 & 500.000 \\
\hline & D. maximus & 20 & $22.000 * \quad(1.7)$ & 1.300 .000 \\
\hline \multirow[b]{2}{*}{60} & $T$. infestans & 20 & 40.000 & 1.560 .000 \\
\hline & D. maximus & 20 & $540.000 *(11.7)$ & 4.600 .000 \\
\hline \multirow{2}{*}{90} & $T$. infestans & 20 & 90.000 & nào feito \\
\hline & D. maximus & 20 & $720.000 *$ & não feito \\
\hline
\end{tabular}

(*) Valores estatisticamente significativos: $\mathrm{X}^{2} 1 \mathrm{~g} 1 \mathrm{p}<0.05$.

O exame realizado demonstrou praticamente não haver diferenças quanto ao número de metacíclicos obtidos de um e outro lote de insetos $(900.000$ e 750.000 por mililitro, respectivamente) confirmando observaçōes anteriores ${ }^{2}$. Também o exame de ninfas de 1 . estádio de D. maximus, separadas em dois lotes (10 em cada), em que um deles ingeriu o dobro de parasitas, ao final de 60 dias apresentaram aproximadamente o mesmo número de tripomastigotas metacíclicos (540.000 e 400.000 por mililitro).

\section{DISCUSSĀO}

A se julgar pelos habitats ou mesmo pela distribuição geográfica das três espécies de triatomineos teria sido viável supor-se o $T$. infestans como um vetor ideal para as duas cepas do $T$. cruzi aqui estudadas, por ser um inseto sabidamente domiciliar e de amplo espectro habitacional, dadas as caracteristicas das diversas regiões geográficas onde é encontrado $^{15}$.

OP. megistus é uma espécie com características de maior exigência climática, encontrando-se tanto em domicílio como no peri-domicilio ${ }^{15}$. Seria considerada talvez como uma espécie com melhores condições de infecção pela cepa Y por ser encontrado no Estado de São Paulo, onde a cepa foi isolada.

O D. maximus, espécie mexicana, cujo habitat é essencialmente silvestre, de região semi-árida 810 , pelos conceitos citados deveria ser a espécie de triatomineo da qual não se poderia esperar bons resul- tados de infecção uma vez que as referidas cepas do T. cruzi são provenientes da região sul do Brasil.

Urdaneta-Morales e Rueda ${ }^{14}$ embora acreditando que a melhor combinação parasita-vetor deva se dar entre individuos de mesma área geográfica, não deixam de assinalar que certamente deva-se levar em conta as diferenças existentes entre os vetores. Os resultados dos experimentos realizados nos levam a admitir que realmente existem tais diferenças e mais, que existem tambem diferenças de interação entre as diversas cepas do $T$. cruz $i$ e os diversos vetores.

Os dados nos levam a acreditar que deva haver realmente um mecanismo de controle de diferenciação do $T$. cruzi nos vetores, como proposto por Sher e Snary ${ }^{13}$, e também um de resistência à infecção o qual se faria manifestar pelo repertorio nutricional existente no trato digestivo destes vetores permitindo ou não um maior desenvolvimento do parasita. Acreditamos que tais fatores se fazem sentir com maior ou menor intensidade, dependendo serem encontrados em maior ou menor volume no trato digestivo dos insetos, devendo-se levar também em consideração a capacidade de reconhecimento por parte do parasita.

Por tal hipótese e também com base nos dados de Alvarenga e Brener ${ }^{1}$, que demonstraram a capacidade de evolução do $T$. cruzi no trato intestinal de triatomíneos na ausência de sangue, concluimos que, 0 $T$. infestans seja uma espécie de triatomineo que apresenta de maneira acentuada, em até $40 \%$ de seus individuos, o fator de resistência à infecção pelo $T$. cruzi uma vez que não foram consideradas significativas as diferenças de perda de infecção desta espécie pelas cepas em estudo, após 60 dias de infecção.

Quanto ao P. megistus consideramos como um vetor apto a receber as duas amostras do $T$. cruzi. Pela hipótese proposta pode-se dizer que trata-se de uma espécie onde o fator regulador de diferenciação se apresenta com caracteristicas semelhantes ao observado em $T$. infestans mas com o fator de resistência à infecção presente de tal forma que permita a implantação das diferentes cepas havendo um baixo indice de perda de infecção. $O$ número de flagelados bem como o percentual de tripomastigotas metaciclicos obtidos a partir de material colhido de $D$. maximus nos levam a admitir que nesta espécie o fator ou fatores que regulam a diferenciação e infecção apresentam-se reduzidos. Esta redução dos fatores permitiria maior diferenciação e menor resistência à implantação dos parasitas. 
Alvarenga NJ, Bronfen E. Interação do Trypanosoma cruzi com diferentes vetores: uso para o xenodiagnóstico. Revista da Sociedade Brasileira de Medicina Tropical 17: 145-149, Jul-Set, 1984

Os dados obtidos sugerem a necessidade de estudos mais aprofundados da fisiologia de triatomíneos associados à relação parasita-hospedeiro.

No que se refere à escolha de insetos para emprego em xenodiagnósticos da doença de Chagas, acreditamos que $D$. maximus seja a espécie indicada, por nossas observações e também as de Barreto e $\mathrm{cols}^{3}$, Marsden e cols ${ }^{8}$ e Cuba e cols ${ }^{7}$ onde são relatadas a capacidade de infecção por diferentes amostras do $T$. cruzi $i$ ainda as facilidades que oferece por sua utilização desde seu primeiro estádio ninfal.

\section{SUMMARY}

Data showing differences in the interaction (differentiation and multiplication) of Trypanosoma cruzi $Y$ and $C L$ strains ("polar strains"), in Triatoma infestans Panstrongylus megistus and Dipetalogaster maximus are presented. It is concluded that with $\mathrm{T}$. infestans $40 \%$ of the individual bugs have accentuated resistance to infection with the $Y$ strain of $\mathrm{T}$. cruzi. $P$. megistus was found to be a good vector to the studied strains presenting good development and differentiation of the parasites with low percentage of rejection of infection. Better development and differentiation is shown by $\mathrm{D}$. maximus which is indicated for xenodiagnosis in Chagas' disease.

The authors suggest the presence of two factors responsible for this vector-parasite interaction: a resistance factor to infection, perhaps related to the presence of nutrients, and also a factor that regulates differentiation to metacyclic forms of $\mathrm{T}$. cruzi

Key words: Trypanosoma cruzi-vector interaction. Xenodiagnosis. Infection resistance factor. Differentiation.

\section{AGRADECIMENTO}

Ao Dr. Philip D. Marsden pelas sugestões apresentadas e à Dra. Vânia A. Soares pela análise estatística.

\section{REFERÊNCIAS BIBLIOGRÁFICAS}

1. Alvarenga NJ. Development of Trypanosoma cruzi in the vector. Congresso Internacional sobre Doença de Chagas, Rio de Janeiro, p. E3-E5, 1979.

2. Alvarenga NJ, Brener Z. Development of Trypanosoma cruz $i$ in the vector in the absence of blood. Acta Tropica 35: 315-317, 1978.
3. Barreto AC, Marsden PD, Cuba CC, Alvarenga NJ. Estudo preliminar sobre o emprego do Dipetalogaster maximus (Uhler, 1894) (Triatominae) na técnica do xenodiagnóstico em forma crônica da doença de Chagas. Revista do Instituto de Medicina Tropical de São Paulo 20: 183-189, 1978.

4. Brener Z. Intraspecific variation in Trypanosoma cruzi: two types of parasitic populations presenting distinct features. PAHO Scientific Publication no 347: 11-21, 1977.

5. Brener Z, Chiari E. Variações morfológicas observadas em diferentes amostras de Trypanosoma cruzi. Revista do Instituto de Medicina Tropical de São Paulo 5: 220224, 1963.

6. Dias E. A comparative study of the susceptibility of four natural vectors to experimental development of Schizotrypanum cruzi. III International Congress of Microbiology, New York, p. 421-422, 1940.

7. Cuba CC, Alvarenga NJ, Barreto AC, Marsden PD, Chiarini C. Nuevos estudios comparativos entre Dipetalogaster maximus y Triatoma infestans en el xenodiagnostico de la infección chagásica crónica humana. Revista do Instituto de Medicina Tropical de Sāo Paulo 20: 145-151, 1978.

8. Marsden PD, Cuba CC, Alvarenga NJ, Barreto AC. Report on a field collection of Dipetalogaster maximus (Hemiptera: Triatominae) (Uhler, 1894). Revista do Instituto de Medicina Tropical de São Paulo 21: 202$206,1979$.

9. Melo RC. Distribuição de parasitas intracelulares em animais inoculados com diferentes cepas de Trypanosoma cruzi. Tese. Universidade Federal de Minas Gerais, 1977.

10. Ryckman RE, Ryckman AE. Epizoothilogy of Trypanosoma cruzi in Southwersten Nort American. Part X. The biosystematics of Dipetalogaster maximus in Mexico (Hemiptera: Reduvidae) (Kinetoplastida: Trypanosomidae). Journal of Medical Entomology 4: 180-188, 1967.

11. Silva LHP, Nussenzweig V. Sobre uma cepa do Trypanosoma cruzi altamente virulenta para o camundongo branco. Folia Clinica et Biologica (São Paulo) 20: 191 $207,1953$.

12. Perlowagora-Szumlewicz A, Müller CA. Studies in search of a suitable experimental insect model for xenodiagnosis of host with Chagas' disease. 1. Comparatives xenodiagnosis with nine triatomine species of animals with acute infection by Trypanosoma cruzi. Memórias do Instituto Oswaldo Cruz 77: 37-53, 1982. 
Alvarenga NJ, Bronfen E. Interação do Trypanosoma cruzi com diferentes vetores: uso para o xenodiagnóstico. Revista da Sociedade Brasileira de Medicina Tropical 17: 145-149, Jul-Set, 1984

13. Sher A, Snary D. Specific inhibition of the morphogenesis of Trypanosoma cruzi by a monoclonal antibody. Nature 300: 639-640, 1982.

14. Urdaneta-Morales S, Rueda IG. A comparative study of the behavior of Venezuelan and Brazilian strains of
Trypanosoma cruz $i$ in the Venezuelan invertebrate host (Rhodnius prolixus). Revista do Instituto de Medicina Tropical de Sào Paulo 19: 241-250, 1977.

15. Zeledón $R$. Los vectores de la enfermedad de Chagas en America. Simposio Internacional sobre Enfermedad de Chagas, Buenos Aires, p. 327-345, 1972. 